\title{
Sliding Test by Using an Apparatus Imitating a Human Finger for Estimating the Tactile Sensation of Cosmetic Foundation*
}

\author{
Keita HORIUCHI** and Ken NAKANO** \\ **Graduate School of Environment and Information Sciences, Yokohama National University, \\ 79-7 Tokiwadai, Hodogaya-ku, Yokohama-shi, Kanagawa, 240-8501 Japan \\ E-mail: keita@davinci.jks.ynu.ac.jp
}

\begin{abstract}
When we acquire tactile sensation, contact and relative motion are necessary between our bodies and objects. This implies that the tactile sensation is produced by friction phenomena. The present paper describes the sliding test for estimating the tactile sensation associated with cosmetic foundation. When the cosmetic foundation is rubbed with an apparatus that imitates a human finger, two types of time-evolving signals - normal and tangential - are obtained as the source of the tactile information. In the sliding test with the apparatus under the frictional condition of humans acquiring the tactile sensation, frictional vibration is observed in the tangential signals, which should be related to the tactile sensation associated with the cosmetic foundation. When a surface with a grooved pattern that imitates fingerprints is used in the sliding test, two types of frictional vibrations are observed. If an adequate sliding velocity is selected, the slight difference between the four types of commercial cosmetic foundations is discriminable by using the signals of these vibrations.
\end{abstract}

Key words: Tactile Sensation, Friction, Free Vibration, Stick-Slip Vibration, Unstable Vibration, Forced Vibration, Cosmetic Foundation

\section{Introduction}

Humans unconsciously obtain circum-information in daily life. This information is crucially important for our secure and comfortable lives. The function is enabled by five senses - auditory, visual, tactile, olfactory, and gustatory.

For acquiring tactile sensation, we have four types of tactile receptors in hairless skin the Merkel disk, the Ruffini ending, the Meissner corpuscle, and the Pacini corpuscle ${ }^{(1)}$. In psychophysical and neurophysiological fields, the responsive properties of tactile receptors have been studied ${ }^{(2)-(7)}$. For example, it is known that the Merkel disk and the Ruffini ending detect the displacement of mechanical stimulation, the Meissner corpuscle detects the velocity, and the Pacini corpuscle detects the acceleration ${ }^{(2)-(4)}$. According to the studies on the response to vibratory stimulation, the Meissner corpuscle detects the stimulations of a lower frequency, e.g., less than $50 \mathrm{~Hz}$, and the Pacini corpuscle detects the stimulations of a higher frequency, e.g., more than $50 \mathrm{~Hz}^{(5)-(7)}$.

The friction phenomena between our bodies and some objects produce the mechanical stimulation. The tactile receptors generate certain electrical signals after detecting the mechanical stimulation. The signals generated by the tactile receptors are transmitted to our brains and analyzed with some appropriate algorithms. By going through the processes, we acquire the tactile sensation. That is, the tactile information should be included in the

${ }^{*}$ Received 9 July, 2007 (No. 07-0291) [DOI: 10.1299/jamdsm.1.726] 
friction signals ${ }^{(8),(9)}$. Therefore, if the friction phenomena are simulated in an apparatus, it should be possible to estimate the tactile sensation by the signals obtained in the system. The production and estimation of the tactile sensation with some apparatuses have been studied $^{(10)-(12)}$; however, many issues with regard to the tactile sensation and friction signals are yet to be clarified.

For example, in the cosmetics industry, the tactile sensation associated with cosmetic foundation has been emphasized as its commodity value, and an improvement in the tactile sensation has been desired. On field sites, questionnaires have been administered to human subjects for the estimation of the tactile sensation associated with cosmetic foundation; however, the estimation method faces some problems not only because many human subjects are required for a valid estimation but also because the estimation results tend to be vague. Therefore, an effective and objective method for the estimation of the tactile sensation associated with the cosmetic foundation is desired.

Therefore, in the present study, friction signals involved in the rubbing of the cosmetic foundation with an apparatus that imitates a human finger have been investigated. In the sliding test with the apparatus under the frictional condition of humans acquiring the tactile sensation, a frictional vibration is observed, which should be related to the tactile sensation associated with the cosmetic foundation. When a surface with a grooved pattern that imitates fingerprints is used in the sliding test, two types of frictional vibrations are observed. If an adequate sliding velocity is selected, the slight difference between four types of commercial cosmetic foundations is discriminable by using the signals of these vibrations.

\section{Experimental details}

\subsection{Apparatus}

An apparatus has been constructed for the purpose described above. It imitates the rubbing action of a human finger, through which the tactile sensation is acquired. The image and schematic diagram of the experimental apparatus are shown in Fig. 1.

In the apparatus, silicon rubber surfaces are employed for imitating human skin. The upper surface is a hemisphere of silicone rubber (diameter: $16 \mathrm{~mm}$, rubber hardness: A20), and the lower surface is a plate of silicone rubber (thickness: $1 \mathrm{~mm}$, rubber hardness: A50).

The upper surface is supported by a double-cantilever spring that allows the movement of the upper surface in the tangential direction (termed the $X$-directional spring), and the double-cantilever spring is supported by another double-cantilever spring that allows the
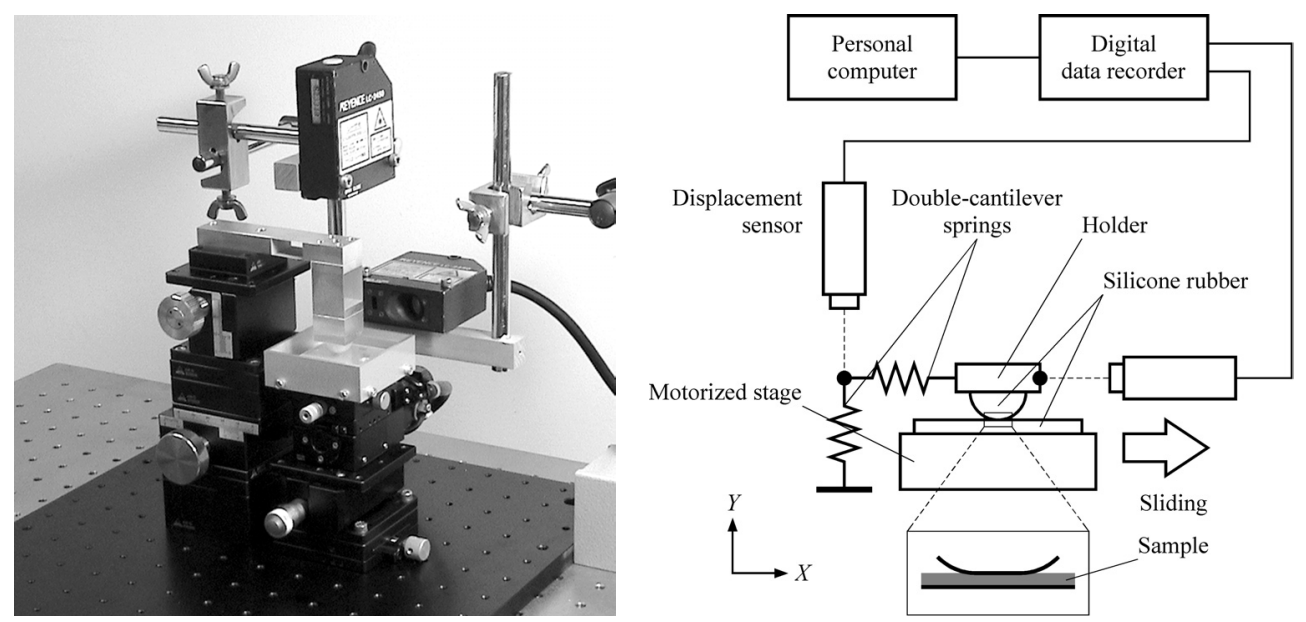

Fig. 1 Image (left) and schematic diagram (right) of the present apparatus. 
movement of the upper surface in the normal direction (termed the $Y$-directional spring). Therefore, the vibration system has two degrees of freedom (2-DOF) in the $X$ and $Y$ directions. The lower surface is mounted on a motorized stage that moves in the $X$ direction. Some manual stages are installed in the apparatus, and they adjust the relative position and direction of the two surfaces.

Three $X$-directional springs have been prepared for investigating the effect of the stiffness, i.e., $685 \mathrm{~N} / \mathrm{m}, 2675 \mathrm{~N} / \mathrm{m}$, and $6518 \mathrm{~N} / \mathrm{m}$. The natural frequency in the $X$ direction is $32 \mathrm{~Hz}$ (for $685 \mathrm{~N} / \mathrm{m}$ ), $59 \mathrm{~Hz}$ (for $2675 \mathrm{~N} / \mathrm{m}$ ), and $90 \mathrm{~Hz}$ (for $6518 \mathrm{~N} / \mathrm{m}$ ). The stiffness of the $Y$-directional springs is $615 \mathrm{~N} / \mathrm{m}$, and the natural frequency in the $Y$ direction is $22 \mathrm{~Hz}$.

When the two surfaces are rubbed against each other, the $X$ - and $Y$-directional springs are deflected. Two laser-type displacement sensors (resolution: $0.5 \mu \mathrm{m}$ ) measure the time evolutions of the spring deflections. The signals from the laser-type displacement sensors are transmitted to a digital data recorder, and the stored data are loaded into a personal computer for analysis.

\subsection{Samples}

Four types of commercial cosmetic foundations were used as samples A-D. They are composed mainly of planer particles (width: $5-50 \mu \mathrm{m}$, thickness: $0.1-0.5 \mu \mathrm{m}$ ), spherical particles (diameter: 0.1-100 $\mu \mathrm{m}$ ), and oil for realizing a good tactile sensation.

According to the questionnaires administered to the human subjects by a cosmetic company using the scoring method, the tactile sensations for samples A and B are of a high quality, that for sample $\mathrm{C}$ is of an average quality, and that for sample $\mathrm{D}$ is of a low quality; however, there is only a slight difference between the qualities of samples A, B, and C.

\subsection{Procedure}

After cleaning the upper and lower surfaces with ethanol, a sample was applied homogeneously on the lower surface by a cosmetic powder-puff. Then, the lower surface was pushed up onto the upper surface by a manual stage in the $Y$ direction. After the contact load was adjusted with the deflection of the $Y$-directional spring, the lower surface was moved in the $X$ direction by the motorized stage, and the signals from the two displacement sensors were stored in the digital data recorder.

The contact load and sliding velocity for imitating the human action of rubbing the surfaces were $0.05-0.25 \mathrm{~N}$ and $1-20 \mathrm{~mm} / \mathrm{s}$, respectively. The sampling frequency of the data recorder was $5000 \mathrm{~Hz}$.

\section{Results and discussion}

\subsection{Time-evolving signals}

Examples of the time evolutions of the spring deflections are shown in Fig. 2, where the stiffness of the $X$-directional spring is $2675 \mathrm{~N} / \mathrm{m}$, the contact load is $0.10 \mathrm{~N}$, and the sliding velocity is $10 \mathrm{~mm} / \mathrm{s}$.

In the sliding test with no sample, as shown in Fig. 2 (left), the $Y$-directional signal is maintained at a constant value corresponding to the constant contact load of $0.10 \mathrm{~N}$. In the $X$-directional signal, the maximal spring deflection is observed when the surfaces start to slide against each other, which is caused by static friction. Then, the spring deflection occurs due to kinetic friction. Such a signal is common in sliding friction.

Further, in the sliding test with sample A, as shown in Fig. 2 (right), the $Y$-directional signal is maintained at the same constant value. On the other hand, the $X$-directional signal changes drastically from the signal with no sample; the spring deflection with the sample is smaller than that with no sample, and a vibration is observed in the signal with the sample. Therefore, it is expected that the tactile information regarding the sample is included in the 
No sample

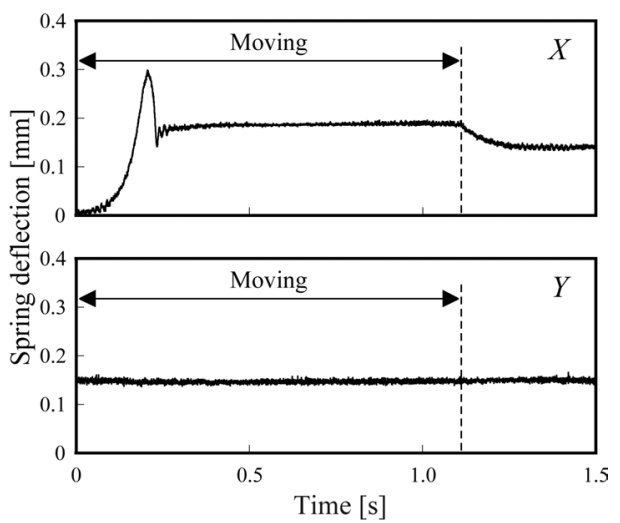

Sample A

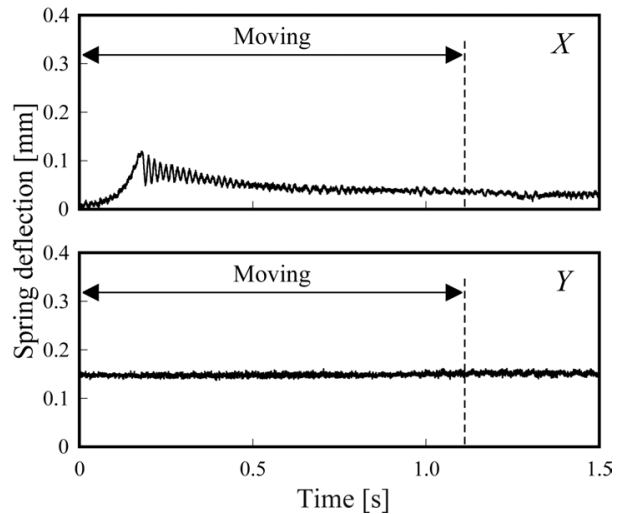

Fig. 2 Time evolutions of spring deflection; stiffness: $2675 \mathrm{~N} / \mathrm{m}$ ( $X$ direction) and $615 \mathrm{~N} / \mathrm{m}$ ( $Y$ direction), contact load: $0.10 \mathrm{~N}$, and sliding velocity: $10 \mathrm{~mm} / \mathrm{s}$.

$X$-directional signal.

\subsection{Static information}

The $X$-directional signals for samples A-D are shown in Fig. 3. In the sliding test with any sample, the spring deflection is smaller than that with no sample, and a vibration is observed in the signals.

As the static information from the signals, static and kinetic friction coefficients were calculated, as shown in Fig. 4, where the maximum static friction was estimated as the product of the $X$-directional stiffness and the maximum spring deflection, and the kinetic friction was estimated as the product of the $X$-directional stiffness and the average of the spring deflection in $0.5-1.0 \mathrm{~s}$. An error bar in the figure shows the standard deviation of five measurements.

The static and kinetic friction coefficients decrease drastically by the application of a sample, which should affect the tactile sensation acquired by humans. However, the difference between the four samples is unclear; at least the friction coefficients of sample A appear to be larger than those of the other samples; however, the questionnaires administered to the human subjects reveal that sample A has the highest quality, as mentioned in section 2.2.

This indicates that another criterion is necessary for estimating the tactile sensation associated with the cosmetic foundation, that is, the dynamic information, e.g., frictional vibration, and not the static information such as friction coefficients.
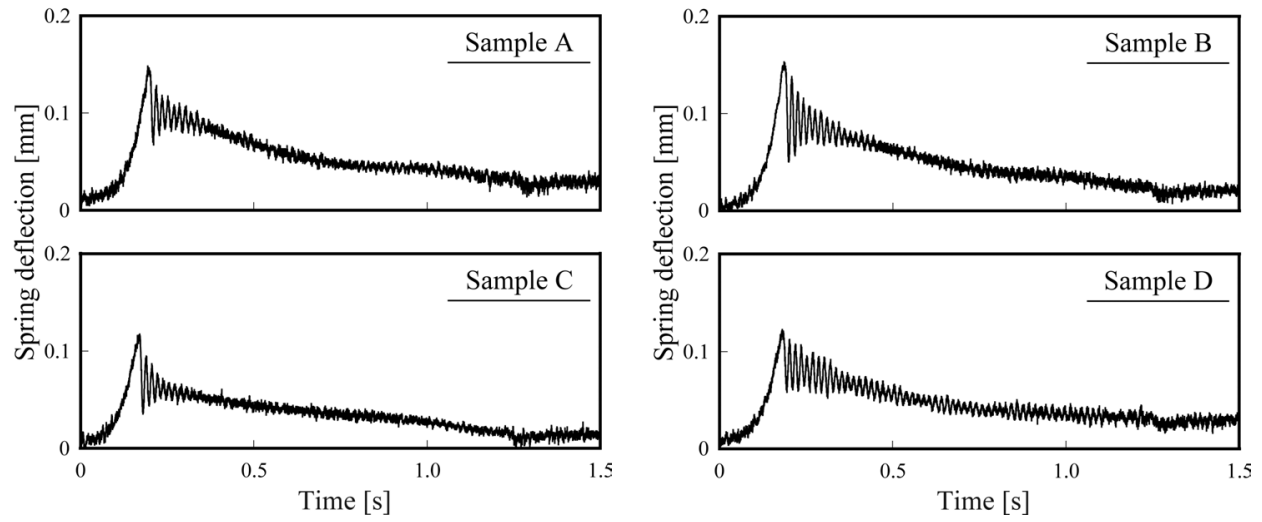

Fig. 3 Time evolutions of spring deflection in $X$ direction; stiffness: $2675 \mathrm{~N} / \mathrm{m}(X$ direction $)$ and $615 \mathrm{~N} / \mathrm{m}$ ( $Y$ direction), contact load: $0.10 \mathrm{~N}$, and sliding velocity: $10 \mathrm{~mm} / \mathrm{s}$. 


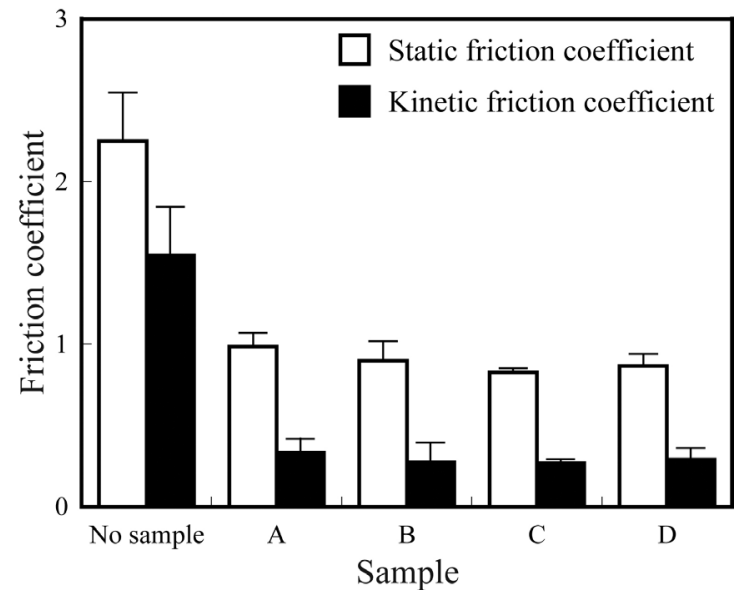

Fig. 4 Static and kinetic friction coefficient; error bar: standard deviation of five measurements.

\subsection{Dynamic information}

The $X$-directional signals are strongly affected by the $X$-directional stiffness. When an $X$-directional spring with the highest stiffness $6518 \mathrm{~N} / \mathrm{m}$ is used, not only the spring deflection but also the frictional vibration decreases, as shown in Fig. 5. Moreover, the signals are so small that they appear to be buried in random noise. On the other hand, with the lowest stiffness $685 \mathrm{~N} / \mathrm{m}$, the spring deflection increases and the frictional vibration is emphasized, as shown in Fig. 6. The effect of the stiffness is consistent with the frictional vibration occurring in 1-DOF systems ${ }^{(13),(14)}$.
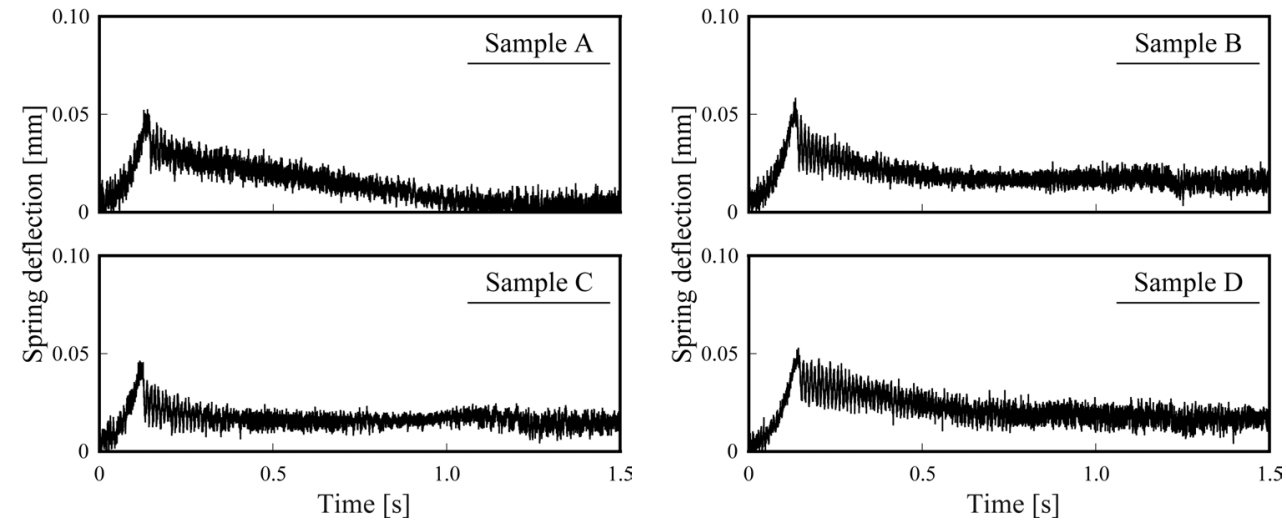

Fig. 5 Time evolutions of spring deflection in $X$ direction under high stiffness; stiffness: $6518 \mathrm{~N} / \mathrm{m}(X$ direction) and $615 \mathrm{~N} / \mathrm{m}$ ( $Y$ direction), contact load: $0.10 \mathrm{~N}$, and sliding velocity: $10 \mathrm{~mm} / \mathrm{s}$.
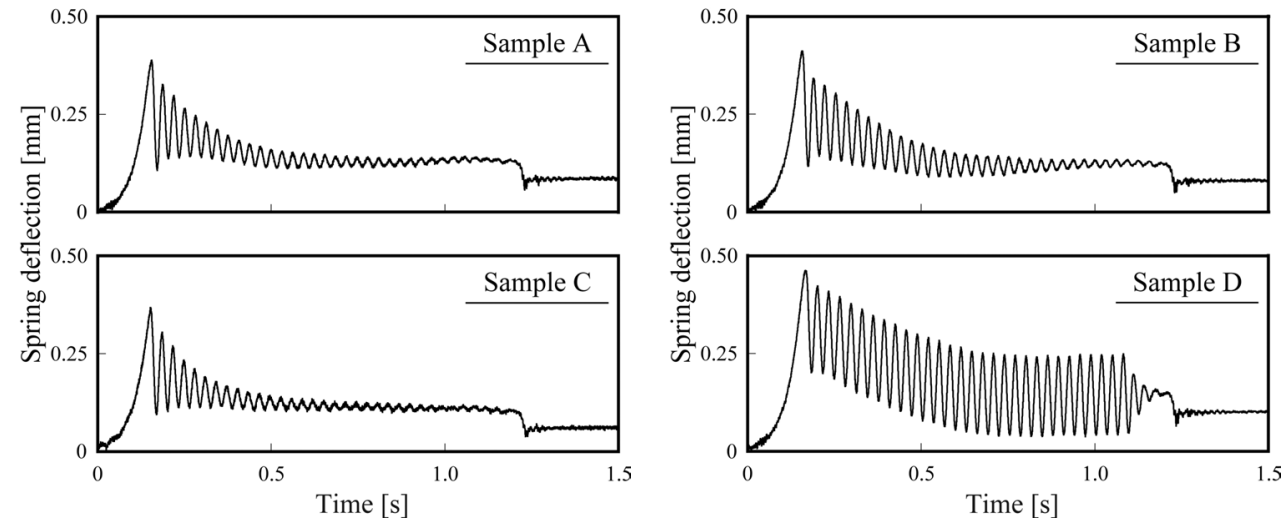

Fig. 6 Time evolutions of spring deflection in $X$ direction under low stiffness; stiffness: $685 \mathrm{~N} / \mathrm{m}(X$ direction) and $615 \mathrm{~N} / \mathrm{m}$ ( $Y$ direction), contact load: $0.10 \mathrm{~N}$, and sliding velocity: $10 \mathrm{~mm} / \mathrm{s}$. 


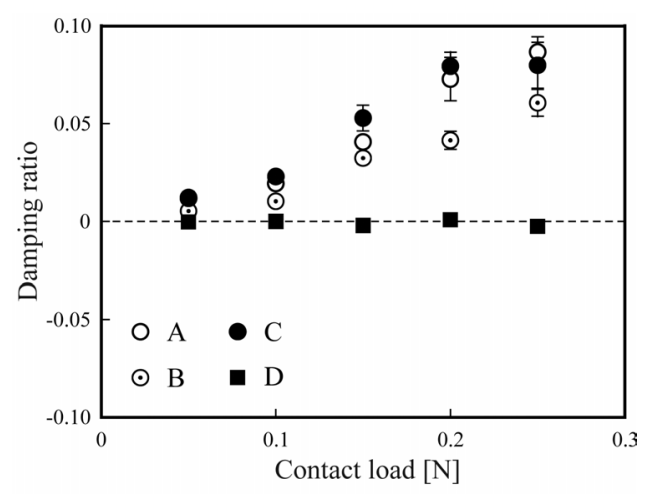

(a)

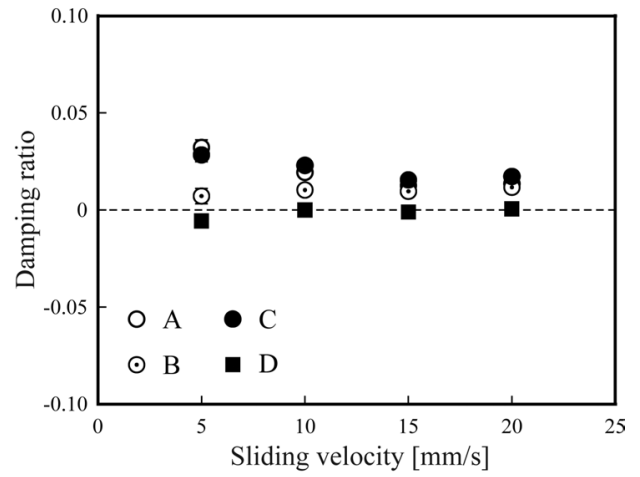

(b)

Fig. 7 Damping ratio of frictional vibration; stiffness: $685 \mathrm{~N} / \mathrm{m}$ ( $X$ direction) and $615 \mathrm{~N} / \mathrm{m}$ ( $Y$ direction) and error bar: standard deviation of five measurements; (a) sliding velocity: $10 \mathrm{~mm} / \mathrm{s}$ and (b) contact load: $0.10 \mathrm{~N}$.

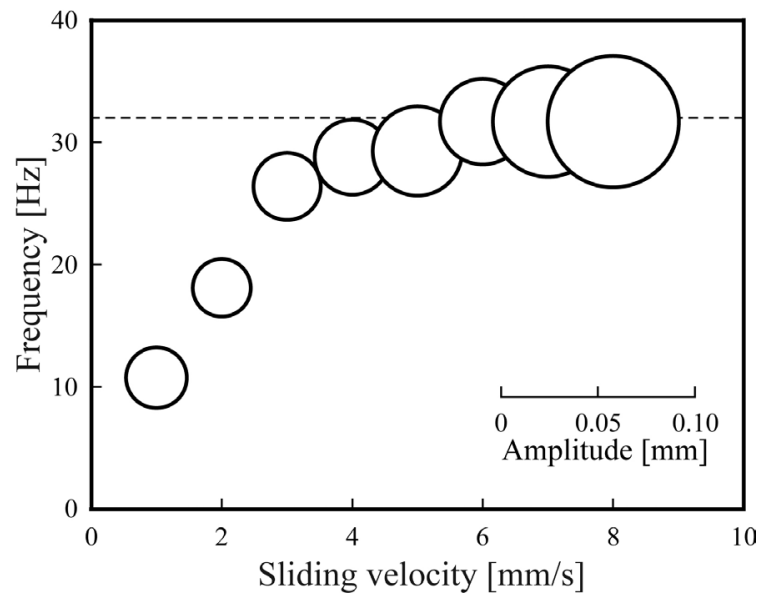

Fig. 8 Frequency and amplitude of frictional vibration; sample: D, stiffness: $685 \mathrm{~N} / \mathrm{m}$ ( $X$ direction) and $615 \mathrm{~N} / \mathrm{m}$ ( $Y$ direction), contact load: $0.20 \mathrm{~N}$, and dashed line: natural frequency in $X$ direction.

The damping ratio was calculated from the decrease in the amplitude of the $X$-directional signals with the variation in the contact load and sliding velocity, where the stiffness in the $X$ direction was the lowest, i.e., $685 \mathrm{~N} / \mathrm{m}$. Figure 7 shows the results.

Under all the conditions in Fig. 7, the damping ratio of sample D is approximately zero, although that of the other samples is positive, that is, an undamped vibration is generated only for sample $\mathrm{D}$, and a damped vibration is generated for the other samples. The difference between the damping ratios of sample $\mathrm{D}$ and the other samples is emphasized under a larger contact load, e.g., under a contact load greater than $0.15 \mathrm{~N}$, as shown in Fig. 7 (a).

Subsequently, the undamped vibration for sample D was analyzed with the fast Fourier transformation of the 2048 discrete data in the $X$-directional signals. Figure 8 shows the change in the frequency and amplitude of the dominant component of the undamped vibration.

When the sliding velocity is lower than $4 \mathrm{~mm} / \mathrm{s}$, the frequency increases, and the amplitude is constant with increasing sliding velocity. On the other hand, for a sliding velocity higher than $6 \mathrm{~mm} / \mathrm{s}$, the frequency is constant corresponding to the natural frequency of $32 \mathrm{~Hz}$, and the amplitude increases with increasing sliding velocity. This trend shows that the former is a stick-slip vibration and the latter is an unstable vibration. Such a transition between the stick-slip vibration and unstable vibration should be generated by the instability of the system ${ }^{(13),(14)}$, and the instability should be determined by the rheology and tribology of the cosmetic foundation. 


\subsection{Grooved-pattern surface for imitating fingerprints}

When humans rub against objects for acquiring a tactile sensation, they tend to vary the sliding velocity continuously. In addition, the surfaces of human fingers have many asperities, i.e., fingerprints. Considering these features of humans, another type of sliding test was conducted, in which the sliding velocity was varied from 0 to $20 \mathrm{~mm} / \mathrm{s}$ with a constant acceleration. Moreover, a grooved pattern that imitates fingerprints was employed on the lower surface perpendicular to the sliding direction; the width of the groove was
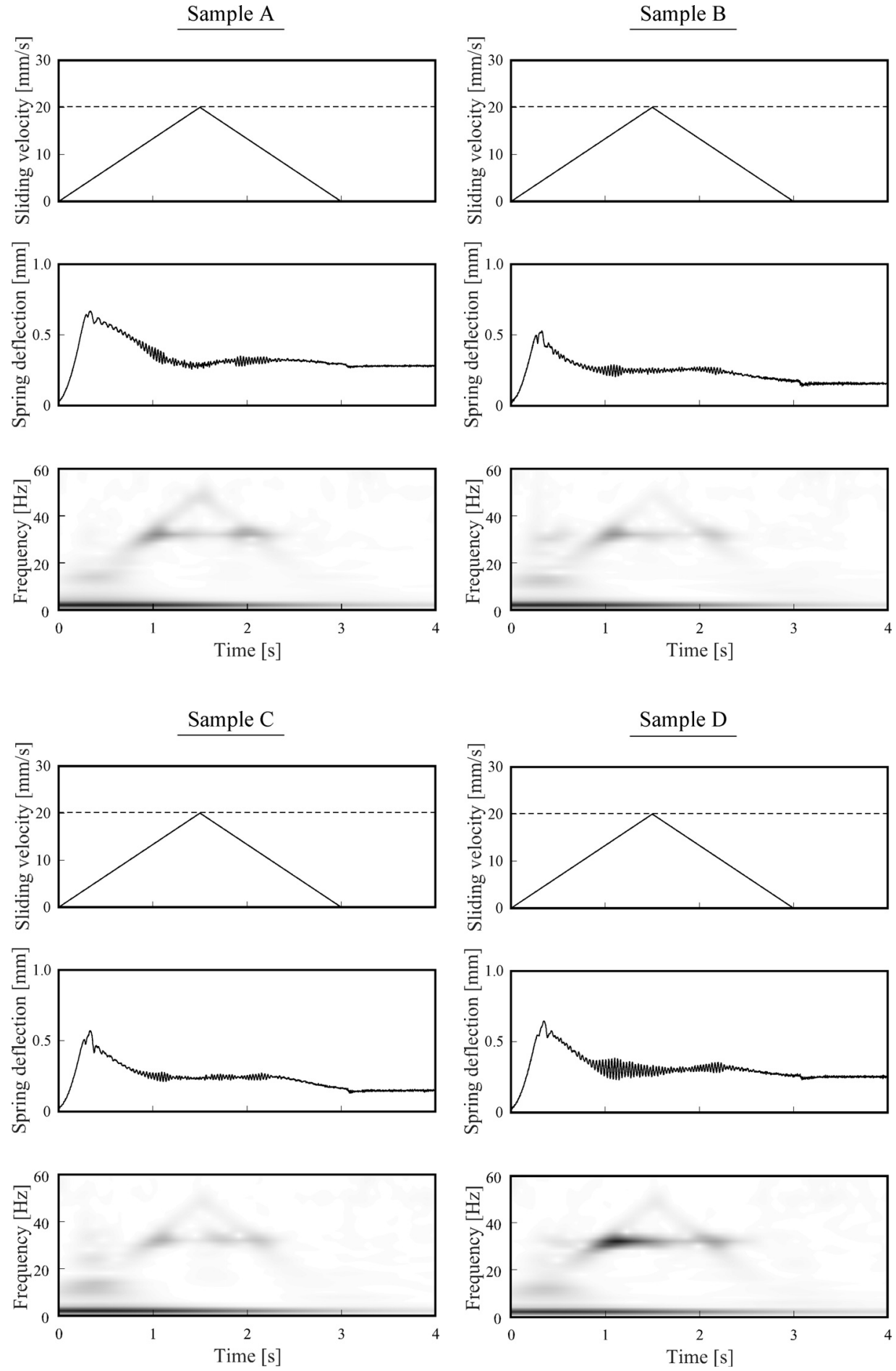

Fig. 9 Time evolutions of sliding velocity (top), spring deflection in $X$ direction (middle), and contour plots obtained from spring deflection with wavelet analysis (bottom) when a grooved-pattern surface is used; stiffness: $685 \mathrm{~N} / \mathrm{m}(X$ direction $)$ and $615 \mathrm{~N} / \mathrm{m}$ ( $Y$ direction $)$ and contact load: $0.20 \mathrm{~N}$. 
$0.2 \mathrm{~mm}$, the depth was $0.1 \mathrm{~mm}$, and the pitch was $0.4 \mathrm{~mm}^{(15)}$.

The time evolutions of the sliding velocity and the spring deflection in the $X$ direction are shown in Fig. 9. The signal of the spring deflection was analyzed by wavelet analysis by using the Gabor wavelet as the mother wavelet. The results are shown in Fig. 9 as contour plots, where the dark area in the plots implies the largeness of the vibration amplitude.

In the contour plots, two types of vibrations are observed. The frequency of a vibration corresponds to the natural frequency of the system, i.e., $32 \mathrm{~Hz}$; it is an unstable vibration. The frequency of the other vibration changes with varying sliding velocity, and the frequency $f$ of this vibration is described by

$$
f=V / D \text {, }
$$

with the sliding velocity $V$ and pitch $D$ of the grooves; it is a forced vibration generated by the grooves ${ }^{(16)}$. We term the former as vibration $I$ and the latter as vibration II.

The sliding velocity increases from 0 to $20 \mathrm{~mm} / \mathrm{s}$ in the period $0-1.5 \mathrm{~s}$. In the period $0-1.0 \mathrm{~s}$, vibration II is generated primarily. At $1.0 \mathrm{~s}$, the frequency of vibration II corresponds to that of vibration I, and the amplitude increases. In the period 1.0-1.5 s, vibrations I and II are observed simultaneously. Further, in the period of decreasing sliding velocity, i.e., the period $1.5-3.0 \mathrm{~s}$, the same trend is recognized; in other words, the most characteristic phenomenon, i.e., the simultaneous generation of the two vibrations, is observed clearly with a sliding velocity of more than $10 \mathrm{~mm} / \mathrm{s}$.

Subsequently, the relationship between the amplitudes of the two vibrations was examined, where the vibration signals were obtained from the experiments for a constant sliding velocity, and they were analyzed with the fast Fourier transformation. The amplitudes of vibration II are plotted against those of vibration I for samples A-D, as shown in Fig. 10.

For a sliding velocity of $10 \mathrm{~mm} / \mathrm{s}$, as shown in Fig. 10 (a), the plotted locations of the four samples overlap. On the other hand, for a sliding velocity of $20 \mathrm{~mm} / \mathrm{s}$, as shown in Fig. 10 (b), the plotted locations of the four samples appear to be separated. This implies that by conducting a sliding test using a grooved-pattern surface under an appropriate frictional condition, the slight difference between the four samples of the commercial cosmetic foundation is discriminable.

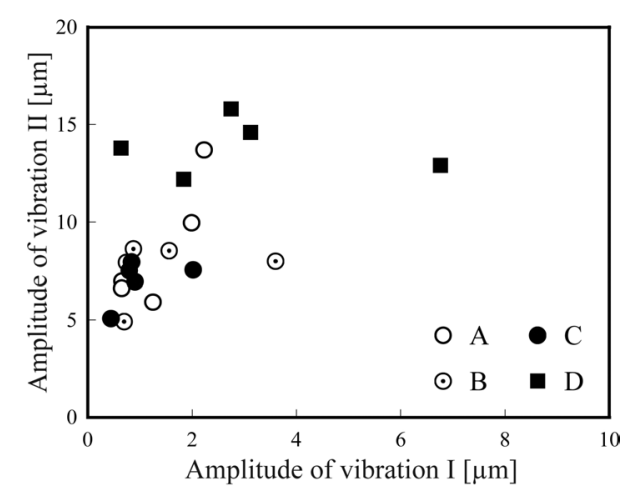

(a)

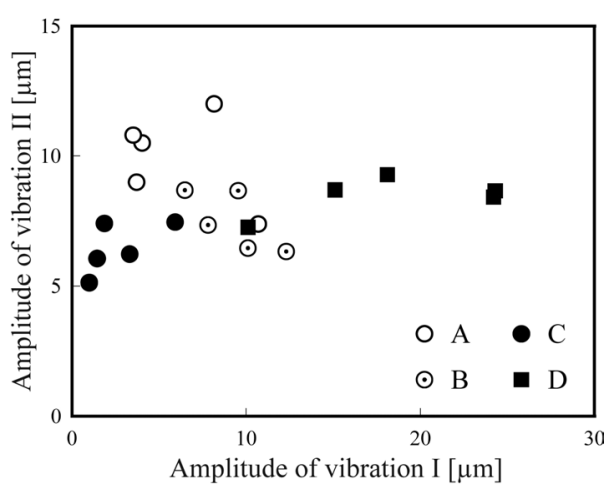

(b)

Fig. 10 Relationship between amplitudes of vibrations I and II; stiffness: $685 \mathrm{~N} / \mathrm{m}$ ( $X$ direction) and 615 $\mathrm{N} / \mathrm{m}$ ( $Y$ direction) and contact load: $0.20 \mathrm{~N}$; (a) sliding velocity: $10 \mathrm{~mm} / \mathrm{s}$ and (b) sliding velocity: $20 \mathrm{~mm} / \mathrm{s}$.

\subsection{Relationship between frictional vibration and tactile sensation}

Using the apparatus mentioned above, the frictional vibration is observed more notably when the stiffness of the $X$-directional spring is low. Considering the softness of the human skin, the friction phenomena detected by the tactile receptors include vibrations as well, 
which should affect the tactile sensation.

As observed previously in Fig. 8, the frequency of the frictional vibration observed in the sliding test with a non-grooved surface was less than $32 \mathrm{~Hz}$, which was determined by the natural frequency of the system. According to a previous study in which the deformation of human fingers was calculated by the finite element analysis, the natural frequency of the shear deformation mode was approximately $20 \mathrm{~Hz}^{(15)}$, which is not very far from the natural frequency of the present apparatus. Therefore, when humans rub against objects for acquiring a tactile sensation, a frictional vibration of less than $20 \mathrm{~Hz}$ is induced in the human skin, which should be detected by the Meissner corpuscles. In addition, it has been known that the Meissner corpuscles play an important role in the recognition of the surface roughness and impalpable asperities ${ }^{(17)}$, which indicates that humans acquire a rough sensation when the Meissner corpuscles are stimulated by the frictional vibration.

In the sliding test with a non-grooved surface, an undamped unstable vibration is measured for sample D; however, a damped free vibration is measured for the other samples, as shown in Fig. 6. It is anticipated that the former will provide a rougher sensation than the latter because the vibration duration of the former is longer than that of the latter. Actually, the result of the questionnaires administered to the human subjects shows that they acquire a rougher sensation for sample D as compared to the other samples. This indicates that the frictional vibration observed in a low-stiffness system contains important information for estimating the tactile sensation.

With regard to damping, the damping ratio of sample B measured by the apparatus is smaller than that of samples A and C, as shown in Fig. 7, which implies that the frictional vibration of sample B is sustained for a slightly longer time than those of samples A and C. Therefore, it is anticipated that humans will acquire a slightly rougher sensation for sample $\mathrm{B}$ than samples A and C; however, the slight difference is not observed in the result of the questionnaires administered to the human subjects. Considering that the tactile sensation of the human subjects inevitably tends to be vague, it is expected that the apparatus will be more sensitive than human sensation.

The sliding test with the grooved-pattern surface generates two types of frictional vibrations - unstable vibration and forced vibration - as shown in Fig. 9. The frequency of the unstable vibration is determined by the natural frequency of the system, and it is expected that the Meissner corpuscles will detect the unstable vibration. On the other hand, as shown in Eq. (1), the frequency of the forced vibration depends on the frictional conditions; therefore, it can become considerably higher than the natural frequency, which indicates that the Pacini corpuscles, and not the Meissner corpuscles, detect the forced vibration under a high sliding velocity and small pitch of the groove. If the two types of vibrations are detected by different types of tactile receptors and recognized as the different information, humans can discriminate the slight difference in the tactile sensation by using the amplitudes of the vibrations, as shown in Fig. 10 (b).

In addition, the results shown in Figs. 9 and 10 indicate the importance of the behavioral and physical characteristics of humans for acquiring the tactile sensation ${ }^{(18)}$. When humans try to recognize the slight difference in the tactile sensation, they tend to rub under various conditions, i.e., changing contact load and sliding velocity. In such actions, they appear to seek a frictional condition in which the slight difference is emphasized maximally; the action is a behavioral characteristic. Moreover, the surfaces of human fingers have fingerprints; it is a physical characteristic, which generates the forced vibration. In the present apparatus, the time-varying sliding velocity imitates the behavioral characteristic, and the groove-pattern surface imitates the physical characteristic. The result shown in Fig. 10 (b) indicates the existence of the frictional condition, in which the slight difference is emphasized maximally by using the behavioral and physical characteristics of humans. 


\section{Conclusions}

Sliding tests have been conducted with an apparatus that imitates a human finger for estimating the tactile sensation associated with of the cosmetic foundation.

(1) Static information (such as static and kinetic friction coefficients) is insufficient for estimating the tactile sensation associated with the cosmetic foundation. Dynamic information (such as the frequency and amplitude of the frictional vibration) is necessary for this purpose.

(2) The apparatus should have a low stiffness for estimating the tactile sensation. A low stiffness emphasizes the frictional vibration, and the rough sensation appears to be related to the duration of the undamped unstable vibration or damped free vibration.

(3) Two types of vibrations - unstable vibration and forced vibration - are generated simultaneously with the grooved-pattern surface that imitates the fingerprints. Under an adequate frictional condition, the slight difference in the four types of commercial cosmetic foundations is discriminable by using the signals of these vibrations.

\section{Acknowledgement}

The authors would like to express their sincere thanks to Kao Corporation for helpful discussions and provision of samples as well as financial support.

\section{References}

(1) Schmidt, R. F., Fundamentals of sensory physiology, Springer-Verlag, (1986).

(2) Johansson, R. S. and Vallbo, A. B., Tactile Sensibility in the Human Hand: Relative and Absolute Densities of Four Types of Mechanoreceptive Units in Glabrous Skin, The Journal of Physiology, Vol.286 (1979), pp.283-300.

(3) Vallbo, A. B. and Johansson, R. S., Properties of Cutaneous Mechanoreceptors in the Human Hand Related to Touch Sensation, Human Neurobiology, Vol.3 (1984), pp.3-14.

(4) Torebjourk, H. E. et al., Intraneural Microstimulation in Man: Its Relation to Specificity of Tactile Sensation, Brain, Vol.110, No.6 (1987), pp.128-140.

(5) Mountcastle, V. B. et al., Detection Thresholds for Stimuli in Humans and Monkeys: Comparison with Threshold Events in Mechanoreceptors Afferent Nerve Fibers Innervating the Monkey Hand, Journal of Neurophysiology, Vol.35 (1972), pp.122-136.

(6) Verrillo, R. T., Psychophysics of Vibrotactile Stimulation, The Journal of the Acoustical Society of America, Vol.77, No.1 (1985), pp.225-232.

(7) Bolanowski, Jr., S. J. et al., Four Channels Mediate the Mechanical Aspects of Touch, The Journal of the Acoustical Society of America, Vol.84, No.5 (1988), pp.1680-1694.

(8) Nakano, K. et al., Discrimination of Particle Diameter by Using Tactile Information (Construction of an Artificial System and Comparison with Human Subject Experiment), Transactions of the Japan Society of Mechanical Engineers, Series C, Vol.73, No.725 (2007), pp.347-353.

(9) Nakano, K. et al., Discrimination of Particle Diameter by Using Tactile Information (Estimation Algorithm of an Artificial System Including the Function of Tactile Receptors), Transactions of the Japan Society of Mechanical Engineers, Series C, Vol.73, No.725 (2007), pp.354-360.

(10) Watanabe, T. and Fukui, S., Control of Tactile Surface-Roughness Sensation Using Ultrasonic Vibration, Transactions of the Japan Society of Mechanical Engineers, Series C, Vol.62, No.596 (1996), pp.1329-1334.

(11) Tanaka, Y., Tanaka, M. and Chonan, S., Measurement of Tactile Sensation Using Sensor System for Collecting Tactile Information, Transactions of the Japan Society of Mechanical Engineers, Series C, Vol.73, No.727 (2007), pp.817-824. 
(12) Kamikawa, Y., Nonomura, Y. and Maeno, T., Relationship between Tribological Characteristics and Perceived Texture When Humans Touch Artificial Skin with/without Lubricants, Transactions of the Japan Society of Mechanical Engineers, Series C, Vol.73, No.730 (2007), pp.1827-1833.

(13) Tadokoro, C. and Nakano, K., A Suppression Method of Friction-Induced Vibration by Controlling Small Dynamic Load, Journal of Japanese Society of Tribologists, Vol.50, No.11 (2005), pp.799-807.

(14) Nakano, K., Two Dimensionless Parameters Controlling the Occurrence of Stick-Slip Motion in a 1-DOF System with Coulomb Friction, Tribology Letters, Vol.24, No.2 (2006), pp.91-98.

(15) Maeno, T. et al., Analysis on Geometry of Human Epidermal Ridges, Transactions of the Japan Society of Mechanical Engineers, Series C, Vol.71, No.701 (2005), pp.245-250.

(16) Nakano, K. et al., Extraction of the Information about Contact Surfaces Based on Frictional-Fluctuation Spectra, Journal of Japanese Society of Tribologists, Vol.50, No.5 (2005), pp.409-415.

(17) Ohka, M. et al., Measurement of Human Tactile Sensation Capability to Discriminate Fine Surface Textures Using a Variable Step-Height Presentation System, Transactions of the Japan Society of Mechanical Engineers, Series C, Vol.64, No.625 (1998), pp.286-292.

(18) Tateishi, T. and Nakano, K., Application of an Array-Structured Sensor and Modified Verification Algorithms to the Personal Authentication System Using Tactile Information, Transactions of the Japan Society of Mechanical Engineers, Series C, Vol.72, No.722 (2006), pp.3213-3220. 